\title{
Affine Transformations and Atlases: Assessing a New Navigation Tool for Knee Arthroplasty
}

\author{
B. $\mathrm{Ma}^{1}$, J.F. Rudan ${ }^{3}$, and R.E. Ellis ${ }^{1,2,3}$ \\ ${ }^{1}$ School of Computing $\quad{ }^{2}$ Mechanical Engineering $\quad{ }^{3}$ Surgery \\ Queen's University at Kingston, Canada \\ ellis@cs.queensu.ca
}

\begin{abstract}
We propose a new guidance paradigm for computer-assisted orthopædic surgery, based on nonrigid registration of a patient to a standard atlas model. Such a method would save costs and time compared to CT-based methods, while being more reliable than current imageless navigation tools. Here, we consider application of this paradigm to knee arthroplasty.

This work reports laboratory results of assessing the simplest nonrigid registration method, which is an affine transformation. Results from CT scans of 15 patients show the method is more repeatable than the standard mechanical guide rods in the frontal plane. The affine transformations also detected a potential difference between osteoarthritic patients and the normal population, which may have implications for the implantation of artificial knee components.
\end{abstract}

\section{Introduction}

Current computer-integrated surgical systems for knee arthroplasty provide navigational guidance relative to CT scans [7,10 12,13,19] or to anatomic features [4,56,8,15] that are identified intraoperatively (such as centers of rotation and anatomic axes). Both methods have notable limitations. The use of CT scans for knee arthroplasty is atypical and introduces extra costs, radiation exposure, and time to produce a patient-specific plan. Systems that require the identification of anatomic features are limited by the accuracy with which these features can be located, and it can be difficult to confirm that the guidance provided is accurate.

We propose that the use of a global deformable shape-based registration may be sufficiently accurate to align a patients' femur to a femur atlas. If our proposition is true, then it would be possible to intraoperatively digitize points from the surfaces of the femur to register the patient to the atlas. A plan defined on the atlas could be applied to the patient without the need for preoperative planning, which simplifies the logistics of delivering care in a clinical setting. Because the guidance is based on shape matching, it would be easy to visually confirm the accuracy of the registration by pointing to distinctive features with a tracked stylus.

Considerable research has been conducted on atlas generation and deformable registration [9], including the mapping of atlas data to patient data for diagnosis and visualization [17]. However, to the best of our knowledge, there have been no published results describing the validation of deformable registration to an atlas for guidance.

\section{A Simple Affine Registration Algorithm}

Our registration is a simple generalization of the ICP algorithm of Besl and McKay [1]. In the original algorithm, ICP repeatedly searches for the set $X$ of model points nearest 
to the transformed data points $P$. Using homogeneous notation and assuming $X$ and $P$ are matrices of column vectors, Horn's method is used to solve for the best rigid transformation $\mathbf{T}_{\text {rigid }}$ :

$$
\min \left\|\mathbf{T}_{\text {rigid }} P-X\right\|_{2}
$$

Since we desire the affine transformation $\mathbf{T}_{\text {affine }}$ we have:

$$
\min \left\|\mathbf{T}_{\text {affine }} P-X\right\|_{2}
$$

which can be solved using any method for optimization of overdetermined linear systems. For the purposes of this study we have chosen to use a least-squares optimization with the $\mathrm{QR}$ decomposition.

Our algorithm has the same guaranteed convergence as the ICP algorithm. The proof of this statement is almost identical to the original proof [1] and is not provided here.

We have not yet explored the problem of how to reliably obtain an initial estimate for this affine registration algorithm. Details of how we proceeded for this study can be found in Section 3 ,

\section{Materials and Methods}

We conducted a retrospective study based on CT scans of patients who enrolled in an approved clinical study of computer-assisted orthopædic surgery and had consented to research use of medical data. Polygonal models of the hip and femur were computed, using an isosurface algorithm, from scans of 15 high tibial osteotomy (HTO) patients. An atlas model was created from the CT scan of a plastic phantom of a normal femur. On the models we identified: the mechanical axis, the anatomic axis, Whiteside and Arima's [18] anteroposterior (AP) axis, the knee center on the surface of the distal femur, and the approximate dimensions of the distal femur in the transverse plane. Studies of the distal femur [16/20] have identified the transepicondylar line (TEL) as an important landmark but we were unable to reliably locate the TEL on some scans.

For each patient model and the atlas we defined the transverse plane to be perpendicular to the mechanical axis. The AP axis was projected into the transverse plane if it was not already perpendicular to the mechanical axis. The frontal plane was defined to be perpendicular to the AP axis, and the sagittal plane was defined to be perpendicular to both the frontal and transverse planes.

To test the baseline accuracy of affine registration for guidance we selected all of the vertices from the lateral, medial, and anterior surfaces of the distal femur, and the vertices from the patellar groove and small regions of the posterior condyles. We approximately matched each femur to the atlas by aligning the knee centers, mechanical axes, and AP axes, and then scaling in the AP and mediolateral (ML) directions. Starting from this initial alignment, we first used ICP to find a rigid registration and then used the modified ICP to find the affine registration. The affine transformation was applied to the patient femurs to assess the registration error relative to the atlas.

For shape-based registration algorithms the value of the registration objective function - usually the root-mean-square (RMS) error for ICP - is a commonly reported measure of error. For comparing a rigid registration to ground truth or a fiducial registration, it is more meaningful to report errors as a rotation and translation about a screw axis [2]. Unfortunately this approach is invalid for affine registration so, in addition to 
computing the RMS error, we also computed registration errors in a manner analogous to the target registration error described by Maurer et al. [11].

Our targets were the knee center and relevant axes of the knee. Reed and Gollish [14] have reported that the potential angular error in the distal femoral cut depends critically on the entry point of the intramedullary rod, and that the correct entry point is located where the anatomic axis exits the distal femur. Because some of our CT scans did not adequately image the distal shaft of the femur, we were unable to define the anatomic axis with reference to the ideal entry point. Instead, we computed the distances between the knee centers on the registered femurs and the atlas instead of the distances between the ideal entry points.

The mechanical axis is a natural target to use because knee arthroplasty attempts to recreate a neutral mechanical axis. We computed the angular alignment errors between the registered and atlas mechanical axes in the frontal and sagittal planes, and also computed the same errors for the respective anatomic axes.

It has been reported [18] that the AP axis is a reliable landmark for alignment in the valgus knee, so we computed the angular alignment errors between the registered and atlas AP axes in the transverse and sagittal planes. Errors were computed in the frame of the atlas. To analyze differences between the phantom and patient atlas results, we used the two-sample t-test with a significance level of $p<0.01$ to test for differences in means and the F-test at a significance level of $p<0.02$ to test for differences in variance.

Even though we used large data sets to perform the registrations, we were still concerned that our results may have been subject to "local-minima trapping", which is a well known drawback of ICP-like algorithms. We performed a stochastic analysis of the sensitivity of the affine-registration process to the initial estimate: starting from our manual alignment, we rotated the patient model by $15^{\circ}$ about a random axis passing through the knee center, and translated by $10 \mathrm{~mm}$ along this axis 1 . We used this new pose as the initial estimate for the registration process. This experiment was repeated 100 times for each patient and we computed the resulting target registration errors.

Finally, we randomly chose one patient to serve as the atlas and registered the remaining patients to the new atlas. We computed errors as for the phantom atlas.

\section{Results}

A visualization of a registered left femur and the atlas axes is shown in Figure 1 that illustrates our error conventions. Notice that if we followed a preoperative plan defined in the atlas coordinate frame, the arthroplasty would result in malalignments in the opposite directions e.g. a varus, hyperextended, internally rotated knee in the case of the illustration.

\subsection{Phantom Atlas}

The RMS error of registration was between $0.79 \mathrm{~mm}$ and $1.39 \mathrm{~mm}$ (mean $1.12 \mathrm{~mm}$ ). The registration errors of the knee center and axes using the phantom atlas are shown in the left column of Figure 2 In the ML and AP directions, the mean knee-center

\footnotetext{
${ }^{1}$ The choice of these values is justified in Section 4.3
} 


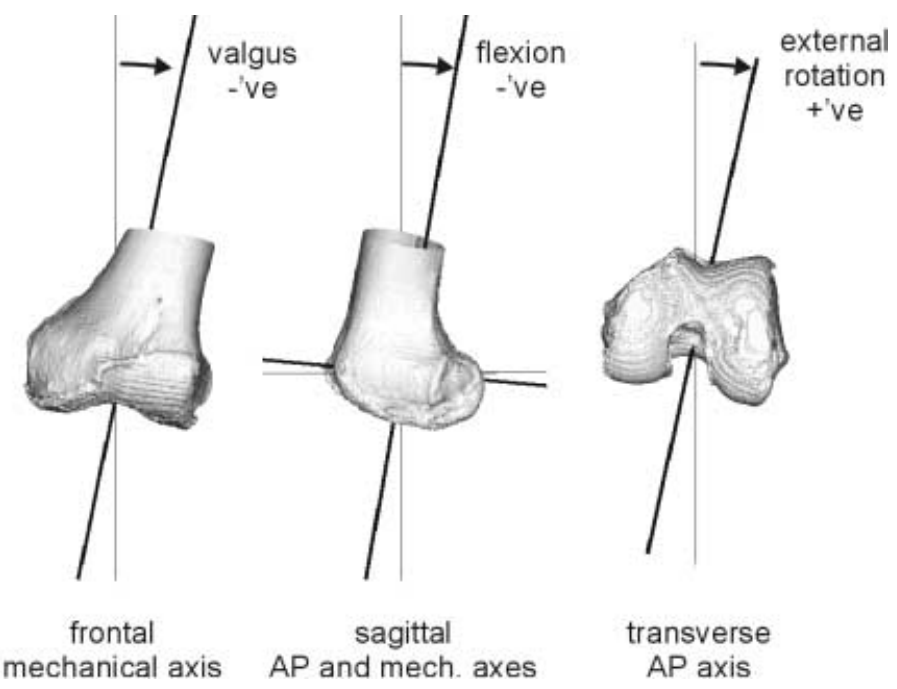

Fig. 1. A convention for measuring axis errors. A transformation that would make an arthroplasty component: in the frontal plane, more varus is positive and more valgus is negative; in the sagittal plane, more extended is positive and more flexed is negative; in the transverse plane, more externally rotated is positive and more internally rotated is negative.

alignment errors were $-0.03 \mathrm{~mm}$ and $-0.18 \mathrm{~mm}$, respectively, with standard deviations (SD) of $1.53 \mathrm{~mm}$ and $2.08 \mathrm{~mm}$. The results for the anatomic and mechanical axes were unusual in that there was a clear trend towards valgus and flexion alignment errors. In the frontal plane, the mean of the mechanical axis alignment errors was $-2.45^{\circ}\left(\mathrm{SD} 2.53^{\circ}\right)$. In the sagittal plane, the mean error was $-4.98^{\circ}\left(\mathrm{SD} 4.60^{\circ}\right)$. For the anatomic axis, the frontal and sagittal plane errors were $-3.32^{\circ}\left(\mathrm{SD} 2.32^{\circ}\right)$ and $-5.29^{\circ}\left(\mathrm{SD} 4.89^{\circ}\right)$. There was a slight trend towards external rotation of the registered femur as indicated by a mean AP axis alignment error in the transverse plane of $0.55^{\circ}$ (SD 5.49 degrees).

\subsection{Patient Atlas}

The registration errors using patient 12 as the atlas are shown in the right column of Figure 2. For the knee center, the alignment errors were $0.31 \mathrm{~mm}(\mathrm{SD} 1.41 \mathrm{~mm})$ and $0.43 \mathrm{~mm}(\mathrm{SD} 2.23 \mathrm{~mm})$ in the ML and AP directions respectively. The F-test values were 1.18 and 0.83 for the ML and AP directions which suggested that there was no significant difference in the variances. The t-test values were -0.6193 and -0.7448 for the ML and AP directions which suggested that there was no significant difference in the means.

The registrations produced a mean neutral alignment of the mechanical axes in the frontal plane (mean $-0.01^{\circ}, \mathrm{SD} 2.15^{\circ}$ ) with a slight trend towards extension alignment error (mean $0.81^{\circ}, \mathrm{SD} 3.81^{\circ}$ ). Variances were smaller than for the phantom atlas but the differences were not significant. (F-test values 1.38 and 1.46). Mean errors were smaller than for the phantom atlas and were significant (t-test values -2.78 and -3.68). Similar conclusions could be drawn for the anatomic axis errors (F-test values 1.53 and 1.39, 
Phantom Atlas
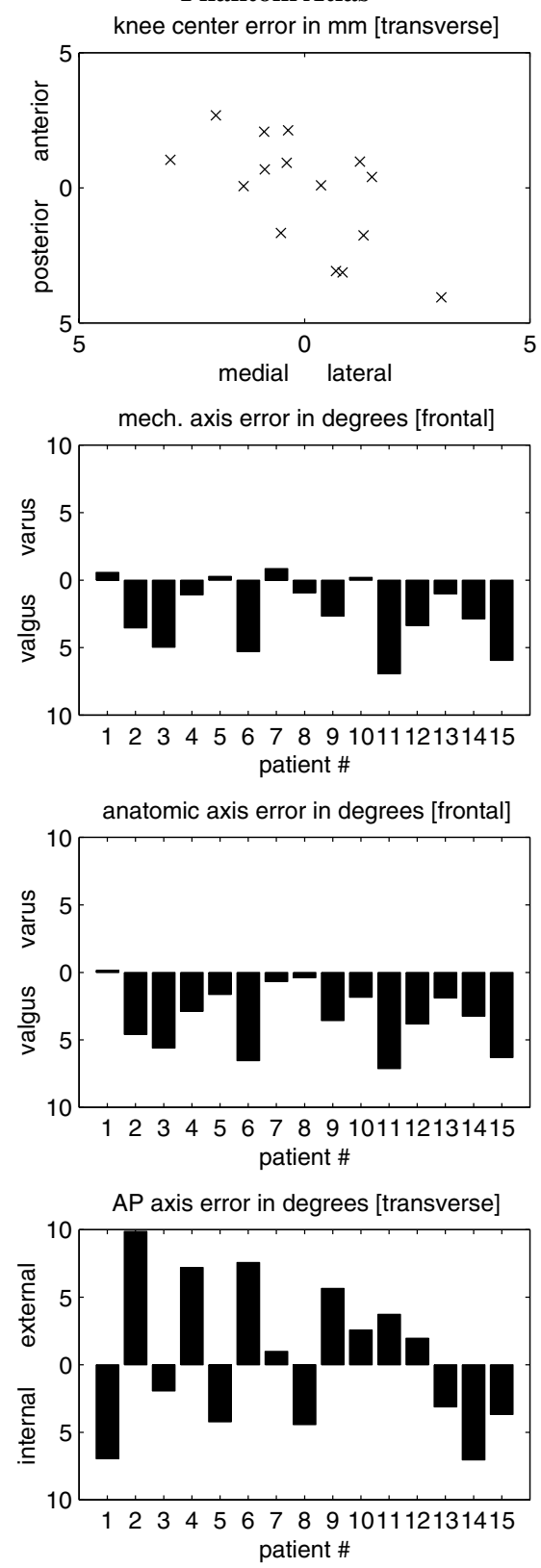

Patient Atlas
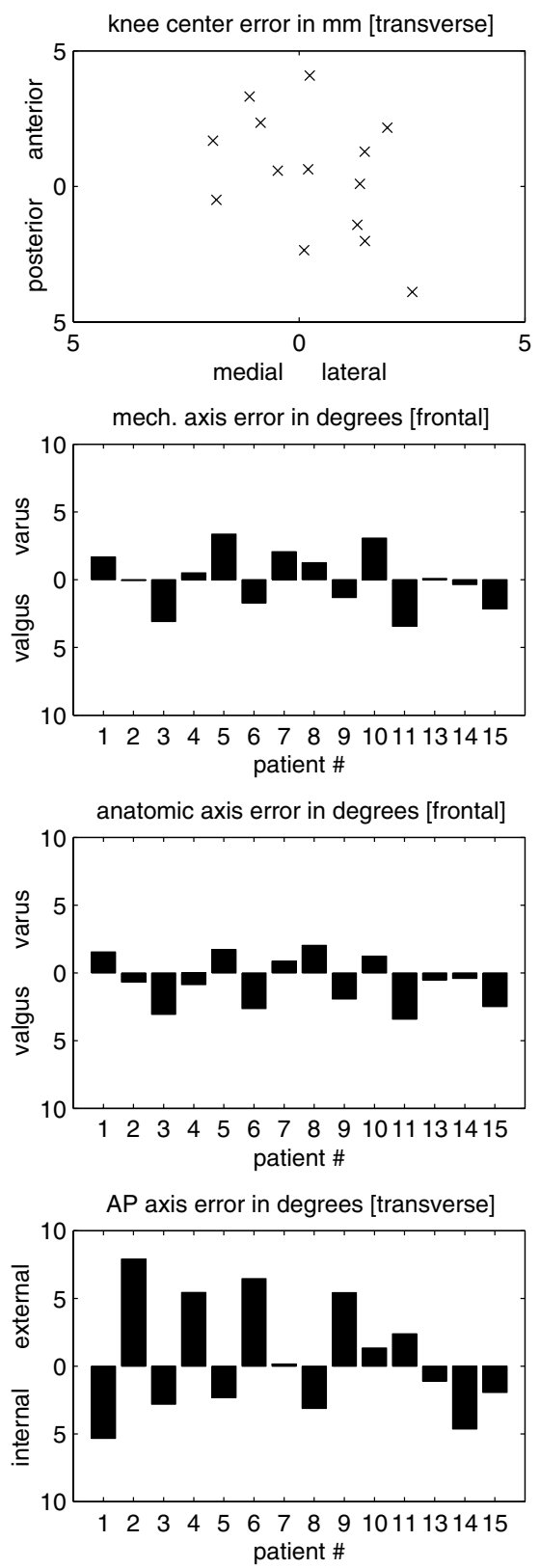

Fig. 2. Alignment errors for the phantom atlas (left) and patient atlas (right). Patient \#12 was used as the atlas and is absent from the patient atlas results. 
t-test values of -3.44 and -4.00 for the frontal and sagittal errors). The frontal and sagittal anatomic axis errors were -0.61 degrees (SD 1.88 $)$ and $1.47^{\circ}\left(\operatorname{SD~} 4.15^{\circ}\right)$.

The AP axis was slightly externally rotated with a mean of 0.57 degrees (SD $4.33^{\circ}$ ). These values were not significantly different from the phantom atlas results (F-test value 1.60 , t-test value -0.01 ).

\subsection{Initial Alignment Sensitivity}

Our results for the phantom atlas showed that the worst alignment errors for the axes were less than $12^{\circ}$ and $5 \mathrm{~mm}$ for the knee center. We arbitrarily increased these values to $15^{\circ}$ and $10 \mathrm{~mm}$ to displace the initial estimates. Since we were investigating the sensitivity to the initial alignment, we computed the standard deviations of the various alignment measurements over the 100 trials for each of the 15 patients. Table 1 shows the largest standard deviations over the 15 patients for each alignment measurement. Given the small values of the maximum standard deviations we were confident that local-minima trapping did not have an effect on our previous results.

Table 1. Initial alignment sensitivity results reported as maximum standard deviations over all patients for each alignment measurement.

\begin{tabular}{|l|c|}
\hline Alignment Measurement & Maximum Standard Deviation \\
\hline knee center, ML & $0.02 \mathrm{~mm}$ \\
knee center, AP & $0.15 \mathrm{~mm}$ \\
\hline mechanical axis, frontal & $0.07^{\circ}$ \\
mechanical axis, sagittal & $0.40^{\circ}$ \\
anatomic axis, frontal & $0.07^{\circ}$ \\
anatomic axis, sagittal & $0.40^{\circ}$ \\
AP axis, frontal & $0.01^{\circ}$ \\
AP axis, sagittal & $0.46^{\circ}$ \\
\hline
\end{tabular}

\section{Discussion}

Our results show that the choice of atlas significantly affects the accuracy of shape-based registration. If the phantom is representative of the normal population, then our results suggest that the shape of the distal femur of an osteoarthritic patient deviates from the norm. This deviation has substantial surgical implications, because a surgeon attempts to align a femoral component so that it is perpendicular to the load-bearing mechanical axis: a malalignment can produce shear forces at the bone interface that can in turn lead to premature failure. If an osteoarthritic patient has a different anatomy than a normal subject, different standards of alignment may need to be considered.

By registering to the shape of the distal femur, we were unable to align the AP axes of the patients and the atlases with good consistency. This is not a surprising result since Whiteside and Arima advocate the use of the AP axis for rotational alignment of the valgus knee because such a knee typically has an abnormally small lateral condyle [18]. Furthermore, Feinstein et al. [3] have found that there is large variation in the alignment of the patellar groove relative to the transepicondylar line in the transverse plane. This 
suggests that affine registration to an atlas derived from a normal subject may not be suitable for determining the rotational alignment of an arthroplasty component.

Our alignment errors as measured in the frontal plane, with a standard deviation of about $2^{\circ}$, compare favorably with the alignment errors induced by intramedullary (IM) guides reported by Reed and Gollish [14]. In their study, the authors' mathematical model predicted up to $3^{\circ}$ valgus malalignment if the insertion point of the IM rod was located at the center of the notch as in the typical textbook description of knee arthroplasty technique. Mechanical guides for unicompartmental arthroplasty are smaller in diameter and less than 1/3 the length of a guide for a total arthroplasty, so the expected alignment errors are much worse (we calculate up to $12^{\circ}$ ).

Limitations of our study include a relatively small number of patients, a very simple registration algorithm, and use of a large amount of data to perform the registration. An interesting extension to our work would be the determination of the minimal amount of data, from surgically accessible regions, needed to reliably perform a deformable registration. Finding a way to acquire or specify the initial registration is also desirable.

This work suggests that it may be practical to perform computer-assisted knee surgery in a completely new way. By comparison with navigation from patient-specific CT scans, deformable registration to an atlas is much less costly, requires no exceptional logistics or labor, and requires no additional radiation to the patient or to the surgical team. By comparison with navigation from anatomical features, deformable registration to an atlas offers the surgeon visual confirmation that (a) a good registration has been achieved, because the surgeon can see the correlation between navigation on the atlas and anatomical features being touched, and (b) visual confirmation that tracking devices attached to the patient or to surgical instruments have not inadvertently moved during surgery. Our results suggest that we can guide a surgeon accurately in the frontal plane, so that conventional instrumentation could be used to complete the procedure.

Affine transformations are a simple first step in exploring the use of deformable registrations for surgical guidance. They are global, and there is no difficulty in transforming simple guidance plans in the atlas (such as a single point, line or plane) to the patient. For guidance that uses more elaborate plans, care must be taken to ensure that all geometric constraints of the operative procedure are met when transforming guidance plans in the atlas to the patient. Future work includes the use of more complex deformable transformations and exploration of the clinical utility of deformable registrations for surgical guidance.

\section{Acknowledgments}

This research was supported in part by Communications and Information Technology Ontario, the Institute for Robotics and Intelligent Systems, the Ontario Research and Development Challenge Fund, and the Natural Sciences and Engineering Research Council of Canada.

\section{References}

1. Paul J. Besl and N. D. McKay. A method for registration of 3-d shapes. IEEE Transactions on Pattern Analysis and Machine Intelligence, 14(2):239-256, February 1992.

2. R. E. Ellis, D. J. Fleet, J. T. Bryant, J. Rudan, and P. Fenton. A method for evaluating ctbased surgical registration. In Jocelyne Troccaz, Eric Grimson, and Ralph Mösges, editors, CVRMed-MRCAS'97, pages 141-150. Springer-Verlag, March 1997. 
3. William K. Feinstein, Philip C. Noble, Emir Kamaric, and Hugh S. Tullos. Anatomic alignment of the patellar groove. Clinical Orthopaedics and Related Research, 331:64-73, 1996.

4. K. B. Inkpen and A. J. Hodgson. Accuracy and repeatability of joint center location in computer-assisted knee surgery. In Medical Image Computing and Computer-Assisted Intervention-MICCAI'99, pages 1072-1079, 1999.

5. K. A. Krackow, L. Serpe, M. J. Phillips, M. Bayers-Thering, and W. M. Mihalkpo. A new technique for determining proper mechanical axis alignment during total knee arthroplasty: Progress toward computer-assisted tka. Orthopedics, 22(7):698-702, July 1999.

6. M. Kunz, F. Langlotz, M. Sati, J. M. Strauss, K. Bernsmann, W. Ruther, and L. P. Nolte. Advanced intraoperative registration of mechanical limb axes for total knee arthroplasty surgery. In CAOS2000, 2000.

7. J. Lea, D. Watkins, A. Mills, M. Peshkin, T. Kienzle III, and S. Stulberg. Registration and immobilization in robot-assisted surgery. Journal of Image Guided Surgery, 1995.

8. F. Leitner, F. Picard, R. Minfelde, H. Schulz, P. Cinquin, and D. Saragaglia. Computer-assisted knee surgical total replacement. In CVRMed-MRCAS'97, pages 629-636, 1997.

9. J. B. A. Maintz and M. A. Viergever. A survey of medical image registration. Medical Image Analysis, 2(1):1-36, 1998.

10. S. Mai, C. Lörke, and W. Siebert. Motivation, realization and first results of robot-assisted total knee arthroplasty. In CAOS2001, 2001.

11. C. Maurer, J. Fitzpatrick, R. Maciunas, and G. Allen. Registration of 3-d images using weighted geometrical features. IEEE TMI, 15(6):836-849, 1996.

12. P. F. La Palombara, M. Fadda, S. Martelli, L. Nofrini, and M. Marcacci. A minimally invasive 3-d data registration protocol for computer and robot assisted total knee arthroplasty. In CVRMed-MRCAS'97, pages 663-672, 1997.

13. F. Picard, A. M. DiGioia, D. Sell, J. E. Moody, B. Jaramaz, C. Nikou, R. S. LaBarca, and T. Levison. Computer-assisted navigation for knee arthroplasty: Intra-operative measurements of alignment and soft-tissue balancing. In CAOS2001, 2001.

14. Stephen C. Reed and Jeffrey Gollish. The accuracy of femoral intramedullary guides in total knee arthroplasty. The Journal of Arthroplasty, 12(6):677-682, 1997.

15. P. Ritschl, R. Fuiko, H. Broers, A. Wurzinger, and W. Berner. Computer-assisted navigation and robot-cutting system for total knee replacement. In CAOS2001, 2001.

16. J. Stiehl and B. Abbott. Morphology of the transepicondylar axis and its application in primary and revision total knee arthroplasty. Journal of Arthrolasty, 6(10):785-789, 1995.

17. P. St-Jean, A. F. Sadikot, L. Collins, D. Clonda, R. Kasrai, A. C. Evans, and T. M. Peters. Automated atlas integration and interactive three-dimensional visualization tools for planning and guidance in functional neurosurgery. IEEE TMI, 15(5):672-680, 1998.

18. Leo A. Whiteside and Junichi Arima. The anteroposterior axis for femoral rotational alignment in valgus total knee arthroplasty. Clinical Orthopaedics, 321:168-172, December 1995.

19. U. Wiesel, A. Lahmer, M. Tenbusch, and M. Boerner. Total knee replacement using the robodoc system. In CAOS2001, 2001.

20. Yuki Yoshioka, David Siu, and T. Derek V. Cooke. The anatomy and functional axes of the femur. The Journal of Bone and Joint Surgery, 69-A(6):873-880, July 1987. 